\title{
AFFINITY AND DIVERSITY INDICES FOR ANOPHELINE IMMATURE FORMS
}

\author{
Lucy NAGM(1), José Francisco LUITGARDS-MOURA(1), César de Souza NEUCAMP(1), Fábio Saito MONTEIRO-DE-BARROS(1), \\ Nildimar Alves HONÓRIO(2), Pantelis TSOURIS(3) \& Maria Goreti ROSA-FREITAS(2,3)
}

\begin{abstract}
SUMMARY
As for the entire Amazon Region, malaria continues to be a major health public problem in Roraima that presented an Annual Parasitic Index of 85.4 in 2005, the highest in Brazil. Information on anopheline breeding sites is an essential component in malaria control strategies. Aiming to contribute to the limited knowledge on anopheline immature forms in Roraima, collections and breeding site observations were performed in 10 breeding sites around the capital city Boa Vista. Collections were carried out in the rainy and dry season periods between April 2004 and January 2005. Breeding sites comprised natural and artificial water reservoirs. A total of 623 immature forms were collected belonging to Anopheles albitarsis s.l., An.triannulatus s.l., An. nuneztovari/ dunhami, An. braziliensis, An. evansae, An. oswaldoi s.l., An. strodei and An. darlingi. An. albitarsis and An. braziliensis were the most frequently found species. Eight larvae of An. darlingi were found in only one breeding site located in the forest. An. triannulatus/ An. nuneztovari and An. albitarsis/An. braziliensis were the pairs of species that mostly occurred together. Both pair of species displayed the highest affinity index what might indicate a high compatibility for the same breeding conditions and/or a synergistic co-occurrence. Species diversity index was higher for the dry season.
\end{abstract}

KEYWORDS: Anophelines; Immatures; Roraima; Brazil.

\section{INTRODUCTION}

As for the entire Amazon, malaria continues to be a major health public problem in Roraima where 31386 malaria cases were registered in 2005. These corresponded to an Annual Parasitic Index (API) of 85.4, the highest in Brazil, hitting the peak of a trend in one of the most malarigeneous states in the latest years $\left(2^{\text {nd }}\right.$ API in 2004 with 71.2 and $3^{\text {rd }}$ API in 2003 with 33.1) $)^{9}$. Information on anopheline immature forms and breeding sites is an essential component in malaria control strategies. In fact, vector control is one of the fundamental and most successful components of the malaria control strategy in the Amazon.

Immature forms and breeding site characterization literature for Roraima is limited ${ }^{3,5,17}$ (National Health Foundation reports-FUNASA Entomological Reports, data not published). In this paper we perform breeding site observations and analyze affinity and diversity indices for the anopheline species collected around Boa Vista during the rainy and dry season of the years 2004-2005.

\section{MATERIAL AND METHODS}

Breeding site characterization: Collections were performed in Boa Vista, Cantá, Bonfim and Alto Alegre municipalities in collections performed in the rainy (April-June 2004) and dry seasons (November 2004-January 2005). Sampling was carried out by dipping three times in 30 points, located one $\mathrm{m}$ apart of each other until a total of 90 samples per site was reached. The total of 90 samples was irrespective of the total breeding site area. A plastic dipper with $350 \mathrm{~mL}$ capacity (standardized by the Pan American Health Organization) was used for this purpose. Breeding sites were photographed and some of their characteristics were observed based on FUNASA's guidelines for immature collection. These characteristics encompassed latitude, longitude, altitude $(\mathrm{m})$, distance of the nearest domicile $(\mathrm{m})$, width $(\mathrm{m})$, length $(\mathrm{m})$, area $\left(\mathrm{km}^{2}\right)$, shading $(\%)$, depth at one $\mathrm{m}$ from the border (m), water flow (strong/medium/weak), type (lagoon, creek, water well, river side, water reservoir, bromeliad, water sewage, marsh, dam, excavation, ground depression, canal, river, flooded land, other), water clarity (clean, cloudy, polluted), vegetation (emergent, underwater, floating), date of the last rain, water temperature, water $\mathrm{pH}$, number of collection points, number of positive collection points, average sampling per point, total number of samples, number and species of anopheline found, number of specimens per instar $\left(1^{\text {st }}-4^{\text {th }}\right.$ larvae and pupae), anopheline species. Land cover type at the collection sites (savannah, alluvial forest, dense forest and transition areas) was also noted.

Laboratory rearing and identification: Larvae were transported to the laboratory (Núcleo Avançado de Vetores, Universidade Federal

Work supported by CNPq-Brazil (Brazilian Council for Science and Technology), part of the undergraduate scholarship of Lucy Nagm (IC-CNPQ-100940/2004-8)

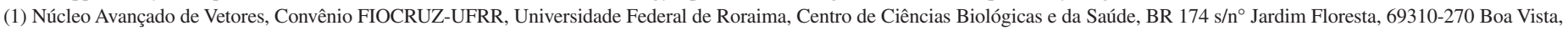
Roraima, RR, Brasil.

(2) Laboratório de Transmissores de Hematozoários, Departamento de Entomologia, Instituto Oswaldo Cruz, FIOCRUZ, Rio de Janeiro, RJ, Brasil.

(3) Freitas-Tsouris Consultants, PO Box 117, Spata-Attikis 19004, Greece.

Correspondence: Dr Maria Goreti Rosa-Freitas, Freitas-Tsouris Consultants, PO Box 117, Spata-Attikis 19004, Greece. E-mail: maria@freitas-tsouris.com 


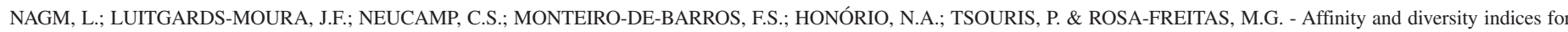
anopheline immature forms. Rev. Inst. Med. trop. S. Paulo, 49(5): 309-316, 2007.

de Roraima-UFRR). First and $2^{\text {nd }}$ instar larvae were reared for identification in the insectary room $\left(26-28{ }^{\circ} \mathrm{C}\right.$ mean room temperature and $70-80 \%$ humidity mean, NAVE-UFRR). Third and $4^{\text {th }}$ instar larvae were identified by the use of taxonomical keys ${ }^{4,10}$. One pupa was reared to adult for identification using the same keys.

Affinity index between species: The index of affinity between pair of anopheline species occurring in the same breeding site was calculated using FAGER \& McGOWAN test ${ }^{8}$ for all species pairs ${ }^{2,7,16,19}$. The test identifies objective groups of recurrent species defined by the strength of their association as $\left[\mathrm{J}(\mathrm{Na} \mathrm{Nb})^{-1 / 2}\right]-\left[1 / 2(\mathrm{Nb})^{1 / 2}\right]^{15}$, where $\mathrm{J}$ is the number of co-occurrences, $\mathrm{Na}$ is the total number of occurrences of species $\mathrm{A}$ alone, $\mathrm{Nb}$ is the total number of occurrences of species $\mathrm{B}$ alone and species are chosen such that $\mathrm{Na} \leq \mathrm{Nb}$. The first term of the equation quantifies the degree of co-occurrence. The second term avoids higher association for rare findings and small sample sizes ${ }^{7,8,16}$. The resulting value provides a quantitative measure of species association. A positive inter-specific threshold value indicative of association was set to values $\geq$ mean plus one standard deviation ${ }^{8,19}$.

Diversity indices: Species diversity for the eight anopheline species in the 10 breeding sites regarding type of land cover and between the rainy and dry seasons were evaluated by using the Shannon diversity index ${ }^{14}$. Shannon's index or species richness is $\left(-\sum_{i=1}^{S} p i \ln p i\right)$ defined by that accounts for abundance and uniformity of the species present. The proportion of species $i$ relative to the total number of species $\left(p_{i}\right)$ is calculated, and then multiplied by the natural logarithm of this proportion $\left(\ln p_{i}\right)$. The resulting product is summed across species and multiplied by -1 . Chi-squared test for statistical significance of all associations was performed.

Meteorological data: Variables recorded were annual average rainfall $(\mathrm{mm})$, temperature $\left({ }^{\circ} \mathrm{C}\right.$, maximum and minimum) and water soil (\%) (Boa Vista Air Force Base and Agritempo ${ }^{1}$ ). The climate presents two distinct seasons, a rainy season between April and November with high rainfall indices during the months of June and July and a dry season from December to March ${ }^{15}$.

\section{RESULTS}

Breeding site characterization: Anopheline immature forms were collected in the municipalities of Boa Vista (six breeding sites), Cantá (one site), Bonfim (one site) and Alto Alegre (two sites) (Fig. 1 and Table 1). Immature form collections took place in the approximate $100 \mathrm{~m}$ peridomiciliary area of houses in permanent breeding sites in four Roraima municipalities (Table 2). Sampling was done in the morning period from 06:00 h to 11:00 h. Number of positive samples per point, number, species and instar of anopheline immature forms found were registered (Table 1). Latitude, longitude and altitude were determined (Table 1, GPS Garmin, Worldwide Geodesic System 1984). All sites had underwater or floating vegetation (Fig. 1). Most breeding sites were of the type water reservoir, had $\geq 50 \%$ of their area shaded and were in savannah areas (Table 2). Water temperature, $\mathrm{pH}$ and other breeding site characteristics were noted for rainy and dry seasons (Table 2). Breeding site variables during the rainy season were water temperature mean $28.9^{\circ} \mathrm{C}(26.5-31.0 \pm 1.6)$ and $\mathrm{pH}$ mean $5.9(5.5-6.5 \pm 0.3)$

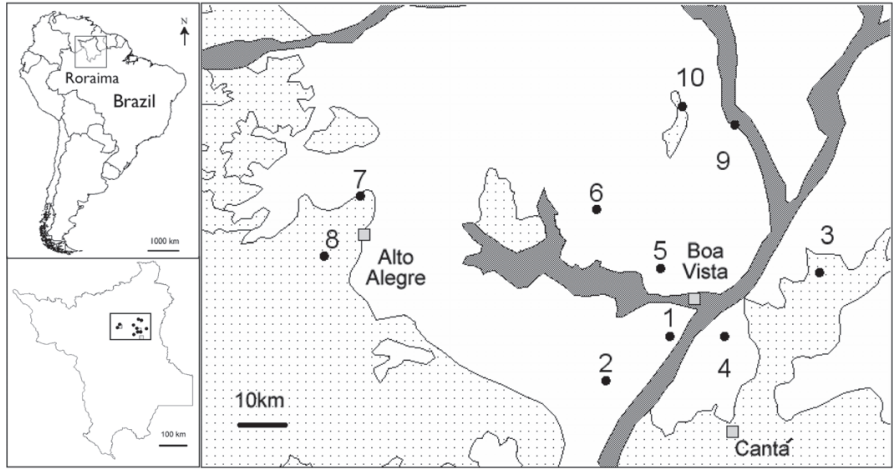

Fig. 1 - South America, Brazil (upper left), Roraima (lower left) and the area (right) where anopheline immature forms were collected. Breeding sites numbered 1 to 10 (localities' names in Table 1). Squares represent the urban centers of the municipalities (Alto Alegre, Boa Vista and Cantá), dotted areas show forest, white areas show savannah and dark grey areas represent alluvial forest.

while during the dry season mean values registered were $29.7^{\circ} \mathrm{C}$ $(28-32.3 \pm 1.4)$ and $5.8(4.9-6.5 \pm 0.6)$ (Table 2).

Laboratory rearing and identification: A total of 623 immature forms were collected belonging to eight distinct species, all from the Nyssorhynchus subgenus. These species were An. albitarsis s.l., An.triannulatus s.l., An. nuneztovari/dunhami, An. braziliensis, An. evansae, An. oswaldoi, An. strodei and An. darlingi (Fig. 2 and Table 2). Anopheline specimens from the Albitarsis, Oswaldoi and Triannulatus species complexes cannot be separated morphologically. These species hereinafter will be cited as An. albitarsis, An. oswaldoi and An.triannulatus. Also by the absence of morphologic variations that allow to ready identification between An. dunhami and An. nuneztovari, the first one was identified as An. nuneztovari by its geographical distribution. An. triannulatus, An. braziliensis and An. albitarsis were the most frequent species found, respectively, while An. evansae, An. oswaldoi and $A n$. darlingi were the least collected (Table 1). During the rainy season, An. triannulatus was the most abundant species (81 larvae) followed by An. albitarsis (65 larvae plus one pupa) and An. nuneztovari (53 larvae) (Fig. 2). In the dry season An. albitarsis was the most abundant species (127), followed by An. braziliensis (85) and An. triannulatus (76) (Fig. 2). An. darlingi was only found in site 10 located in the forest in the rainy season (one specimen), and in the dry season (seven specimens) (Tables 1 and 2) (Fig. 1 and 2). An. albitarsis was found in all 10 sites (Table 1 and Fig. 2).

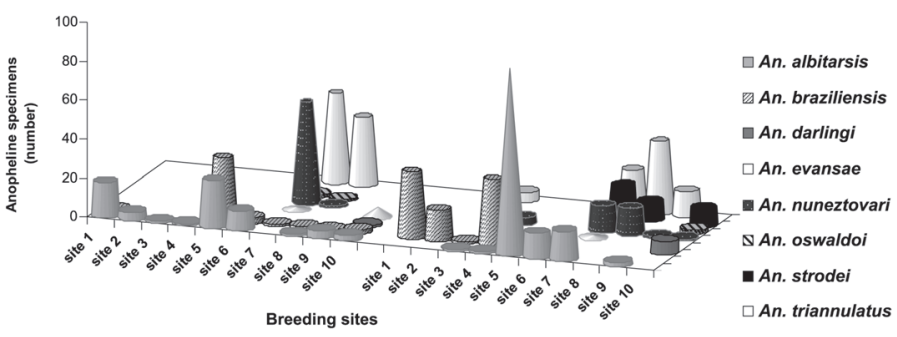

Fig. 2 - Anopheline species and number of specimens for 10 breeding sites during the rainy and dry seasons 2004-2005, Roraima, Brazil. 


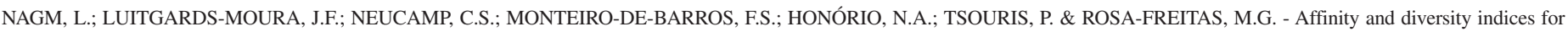
anopheline immature forms. Rev. Inst. Med. trop. S. Paulo, 49(5): 309-316, 2007.

Table 1

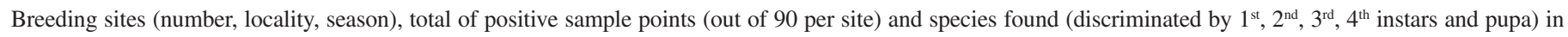
Roraima, Brazil, 2004-2005

\begin{tabular}{|c|c|c|c|c|c|c|c|c|c|c|}
\hline $\begin{array}{l}\text { Breeding } \\
\text { site }\end{array}$ & $\begin{array}{l}\text { Locality } \\
\text { (Latitude, longitude, altitude-m) }\end{array}$ & Season & $\begin{array}{l}\text { Positive } \\
\text { points }\end{array}$ & Species found & $1^{\text {st }}$ & & $3^{\text {rd }}$ & $4^{\text {th }}$ & Pupa & Total \\
\hline 1 & $\begin{array}{l}\text { Centenário, Boa Vista } \\
\left(02^{\circ} 47^{\prime} 34^{\prime}, 60^{\circ} 43^{\prime} 04^{\prime}, 80\right)\end{array}$ & $\begin{array}{l}\text { Rainy } \\
\text { dry }\end{array}$ & $\begin{array}{l}15 \\
19\end{array}$ & $\begin{array}{l}\text { An. albitarsis } \\
\text { An. braziliensis } \\
\text { An. braziliensis }\end{array}$ & $\begin{array}{c}1 \\
0 \\
10\end{array}$ & $\begin{array}{c}13 \\
1 \\
14\end{array}$ & $\begin{array}{l}4 \\
0 \\
8\end{array}$ & $\begin{array}{l}0 \\
0 \\
2\end{array}$ & $\begin{array}{l}0 \\
0 \\
0\end{array}$ & $\begin{array}{c}18 \\
1 \\
34\end{array}$ \\
\hline 2 & $\begin{array}{l}\text { BR-174 Km 21, Boa Vista } \\
\left(02^{\circ} 42^{\prime} 26^{\prime}, 60^{\circ} 50^{\prime} 26^{\prime}, 87\right)\end{array}$ & $\begin{array}{l}\text { rainy } \\
\text { dry }\end{array}$ & $\begin{array}{c}4 \\
11\end{array}$ & $\begin{array}{l}\text { An. albitarsis } \\
\text { An. braziliensis }\end{array}$ & $\begin{array}{l}4 \\
6\end{array}$ & $\begin{array}{l}0 \\
3\end{array}$ & $\begin{array}{l}0 \\
5\end{array}$ & $\begin{array}{l}0 \\
2\end{array}$ & $\begin{array}{l}0 \\
0\end{array}$ & $\begin{array}{c}4 \\
16\end{array}$ \\
\hline 3 & $\begin{array}{l}\text { BR-401, São Francisco Village, } \\
\text { Bonfim } \\
\left(02^{\circ} 54^{\prime} 50^{\prime \prime}, 60^{\circ} 25^{\prime} 41^{\prime \prime}, 100\right)\end{array}$ & $\begin{array}{l}\text { rainy } \\
\text { dry }\end{array}$ & $\begin{array}{c}1 \\
13\end{array}$ & $\begin{array}{l}\text { An. albitarsis } \\
\text { An. albitarsis } \\
\text { An. braziliensis } \\
\text { An. triannulatus } \\
\text { An. nuneztovari }\end{array}$ & $\begin{array}{l}0 \\
0 \\
0 \\
3 \\
1\end{array}$ & $\begin{array}{l}0 \\
1 \\
1 \\
1 \\
3\end{array}$ & $\begin{array}{l}1 \\
0 \\
0 \\
1 \\
0\end{array}$ & $\begin{array}{l}0 \\
0 \\
0 \\
0 \\
0\end{array}$ & $\begin{array}{l}0 \\
0 \\
0 \\
0 \\
0\end{array}$ & $\begin{array}{l}1 \\
1 \\
1 \\
5 \\
4\end{array}$ \\
\hline 4 & $\begin{array}{l}\text { BR-401, Santa Cecília, Cantá } \\
\left(02^{\circ} 47^{\prime} 34^{\prime}, 60^{\circ} 36^{\prime} 43^{\prime \prime}, 84\right)\end{array}$ & $\begin{array}{l}\text { rainy } \\
\text { dry }\end{array}$ & $\begin{array}{c}1 \\
32\end{array}$ & $\begin{array}{l}\text { An. albitarsis } \\
\text { An. albitarsis } \\
\text { An. braziliensis } \\
\text { An. nuneztovari }\end{array}$ & $\begin{array}{c}0 \\
0 \\
13 \\
4\end{array}$ & $\begin{array}{c}1 \\
1 \\
17 \\
0\end{array}$ & $\begin{array}{l}0 \\
0 \\
2 \\
0\end{array}$ & $\begin{array}{l}0 \\
0 \\
2 \\
0\end{array}$ & $\begin{array}{l}0 \\
0 \\
0 \\
0\end{array}$ & $\begin{array}{c}1 \\
1 \\
34 \\
4\end{array}$ \\
\hline 5 & $\begin{array}{l}\text { Monte Cristo III, Refazenda Farm, } \\
\text { Boa Vista } \\
\left(02^{\circ} 55^{\prime} 18^{\prime \prime}, 60^{\circ} 44^{\prime} 08^{\prime \prime}, 80\right)\end{array}$ & $\begin{array}{l}\text { rainy } \\
\text { dry }\end{array}$ & $\begin{array}{l}33 \\
37\end{array}$ & $\begin{array}{l}\text { An. albitarsis } \\
\text { An. braziliensis } \\
\text { An. albitarsis }\end{array}$ & $\begin{array}{l}15 \\
17 \\
16\end{array}$ & $\begin{array}{c}7 \\
13 \\
43\end{array}$ & $\begin{array}{c}1 \\
2 \\
18\end{array}$ & $\begin{array}{c}0 \\
0 \\
18\end{array}$ & $\begin{array}{l}1 \\
0 \\
0\end{array}$ & $\begin{array}{l}24 \\
32 \\
95\end{array}$ \\
\hline 6 & $\begin{array}{l}\text { Bamerindus Farm, N.Sra. Aparecida } \\
\text { Ranch, Boa Vista } \\
\left(03^{\circ} 02^{\prime} 04^{\prime \prime}, 60^{\circ} 51^{\prime} 31^{\prime \prime}, 99\right)\end{array}$ & $\begin{array}{l}\text { rainy } \\
\text { dry }\end{array}$ & $\begin{array}{l}12 \\
10\end{array}$ & $\begin{array}{l}\text { An. albitarsis } \\
\text { An. braziliensis } \\
\text { An. albitarsis }\end{array}$ & $\begin{array}{l}4 \\
2 \\
2\end{array}$ & $\begin{array}{l}1 \\
1 \\
5\end{array}$ & $\begin{array}{l}1 \\
0 \\
3\end{array}$ & $\begin{array}{l}4 \\
0 \\
3\end{array}$ & $\begin{array}{l}0 \\
0 \\
0\end{array}$ & $\begin{array}{c}10 \\
3 \\
13\end{array}$ \\
\hline 7 & $\begin{array}{l}\text { RR-205, São Sebastião Ranch, } \\
\text { Alto Alegre } \\
\left(03^{\circ} 03 ’ 37^{\prime}, 61^{\circ} 18^{\prime} 45^{\prime}, 115\right)\end{array}$ & rainy & 40 & $\begin{array}{l}\text { An. braziliensis } \\
\text { An. triannulatus } \\
\text { An. nuneztovari } \\
\text { An. oswaldoi } \\
\text { An. evansae } \\
\text { An. albitarsis } \\
\text { An. triannulatus } \\
\text { An. nuneztovari } \\
\text { An. evansae } \\
\text { An. strodei }\end{array}$ & $\begin{array}{c}1 \\
37 \\
33 \\
2 \\
0 \\
10 \\
14 \\
13 \\
0 \\
14\end{array}$ & $\begin{array}{l}0 \\
3 \\
5 \\
0 \\
0 \\
4 \\
4 \\
1 \\
1 \\
2\end{array}$ & $\begin{array}{c}0 \\
5 \\
12 \\
0 \\
1 \\
1 \\
2 \\
0 \\
0 \\
1\end{array}$ & $\begin{array}{l}0 \\
1 \\
2 \\
0 \\
0 \\
0 \\
1 \\
0 \\
0 \\
0\end{array}$ & $\begin{array}{l}0 \\
0 \\
0 \\
0 \\
0 \\
0 \\
0 \\
0 \\
0 \\
0\end{array}$ & $\begin{array}{c}1 \\
46 \\
52 \\
2 \\
1 \\
15 \\
21 \\
14 \\
1 \\
17\end{array}$ \\
\hline 8 & $\begin{array}{l}\text { RR-343, São José Farm, } \\
\text { Alto Alegre } \\
\left(02^{\circ} 56^{\prime} 42^{\prime}, 6^{\circ} 22^{\prime} 52^{\prime},, 100\right)\end{array}$ & dry & 48 & $\begin{array}{l}\text { An. albitarsis } \\
\text { An. braziliensis } \\
\text { An. triannulatus } \\
\text { An. nuneztovari } \\
\text { An. oswaldoi } \\
\text { An. triannulatus } \\
\text { An. nuneztovari } \\
\text { An. strodei }\end{array}$ & $\begin{array}{c}0 \\
0 \\
13 \\
0 \\
0 \\
8 \\
2 \\
6\end{array}$ & $\begin{array}{c}0 \\
0 \\
15 \\
1 \\
1 \\
22 \\
10 \\
0\end{array}$ & $\begin{array}{l}0 \\
1 \\
5 \\
0 \\
0 \\
5 \\
1 \\
2\end{array}$ & $\begin{array}{l}1 \\
0 \\
2 \\
0 \\
0 \\
2 \\
1 \\
2\end{array}$ & $\begin{array}{l}0 \\
0 \\
0 \\
0 \\
0 \\
0 \\
0 \\
0\end{array}$ & $\begin{array}{c}1 \\
1 \\
35 \\
1 \\
1 \\
37 \\
14 \\
10\end{array}$ \\
\hline 9 & $\begin{array}{l}\text { Passarão, Boa Vista } \\
\left(03^{\circ} 11^{\prime} 57^{\prime}, 60^{\circ} 35^{\prime} 30^{\prime}, 91\right)\end{array}$ & $\begin{array}{l}\text { rainy } \\
\text { dry }\end{array}$ & $\begin{array}{l}4 \\
13\end{array}$ & $\begin{array}{l}\text { An. albitarsis } \\
\text { An. braziliensis } \\
\text { An. albitarsis } \\
\text { An. triannulatus } \\
\text { An. nuneztovari }\end{array}$ & $\begin{array}{l}1 \\
0 \\
0 \\
3 \\
0\end{array}$ & $\begin{array}{l}1 \\
0 \\
1 \\
6 \\
0\end{array}$ & $\begin{array}{l}0 \\
1 \\
0 \\
2 \\
1\end{array}$ & $\begin{array}{l}2 \\
0 \\
1 \\
2 \\
0\end{array}$ & $\begin{array}{l}0 \\
0 \\
0 \\
0 \\
0\end{array}$ & $\begin{array}{c}4 \\
1 \\
2 \\
13 \\
1\end{array}$ \\
\hline 10 & $\begin{array}{l}\text { Serra da Moça Creek, Boa Vista } \\
\left(03^{\circ} 14^{\prime} 01^{\prime \prime}, 60^{\circ} 41^{\prime} 38^{\prime \prime}, 117\right)\end{array}$ & dry & 10 & $\begin{array}{l}\text { An. darlingi } \\
\text { An. albitarsis } \\
\text { An. braziliensis } \\
\text { An. evansae } \\
\text { An. darlingi } \\
\text { An. nuneztovari } \\
\text { An. oswaldoi } \\
\text { An. strodei }\end{array}$ & $\begin{array}{l}1 \\
3 \\
1 \\
0 \\
0 \\
0 \\
1 \\
0\end{array}$ & $\begin{array}{l}0 \\
0 \\
1 \\
2 \\
2 \\
0 \\
0 \\
6\end{array}$ & $\begin{array}{l}0 \\
0 \\
0 \\
2 \\
2 \\
0 \\
1 \\
3\end{array}$ & $\begin{array}{l}0 \\
0 \\
0 \\
1 \\
3 \\
1 \\
0 \\
0\end{array}$ & $\begin{array}{l}0 \\
0 \\
0 \\
0 \\
0 \\
0 \\
0 \\
0\end{array}$ & $\begin{array}{l}1 \\
3 \\
2 \\
5 \\
7 \\
1 \\
2 \\
9\end{array}$ \\
\hline TOTAL & 10 & & 380 & 8 & 261 & 214 & 94 & 53 & 1 & 623 \\
\hline
\end{tabular}




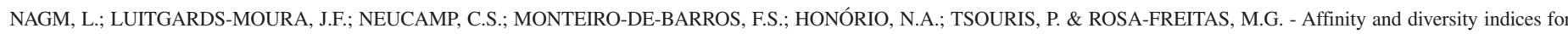
anopheline immature forms. Rev. Inst. Med. trop. S. Paulo, 49(5): 309-316, 2007.

Table 2

Characteristics of 10 permanent breeding sites in four municipalities of Roraima, 2004-2005

\begin{tabular}{|c|c|c|c|c|c|c|c|c|c|c|c|c|c|c|c|}
\hline \multirow[t]{2}{*}{$\begin{array}{l}\text { Breeding } \\
\text { site }\end{array}$} & \multirow[t]{2}{*}{ Type } & \multirow[t]{2}{*}{$\begin{array}{l}\text { Surrounding } \\
\text { vegetation }\end{array}$} & \multirow[t]{2}{*}{$\begin{array}{l}\text { Water } \\
\text { speed }\end{array}$} & \multirow[t]{2}{*}{$\begin{array}{l}\text { Water } \\
\text { clarity }\end{array}$} & \multirow[t]{2}{*}{$\begin{array}{c}\text { Water } \\
\text { vegetation }\end{array}$} & \multirow{2}{*}{$\begin{array}{l}\text { Nearest } \\
\text { domicile } \\
\text { (m) }\end{array}$} & \multicolumn{3}{|c|}{$\begin{array}{l}\text { Approximate } \\
\text { dimensions }\end{array}$} & \multirow[t]{2}{*}{$\begin{array}{l}\text { Shading } \\
(\%)\end{array}$} & \multirow{2}{*}{$\begin{array}{l}\text { Depth } \\
\text { at } 1 \mathrm{~m} \\
(\mathrm{~m})\end{array}$} & \multicolumn{2}{|c|}{$\begin{array}{c}\text { Water } \\
\text { temperature }\left({ }^{\circ} \mathrm{C}\right)\end{array}$} & \multicolumn{2}{|c|}{$\mathrm{pH}$} \\
\hline & & & & & & & $\begin{array}{l}\text { Width } \\
(\mathrm{m})\end{array}$ & $\begin{array}{l}\text { Length } \\
\text { (m) }\end{array}$ & $\begin{array}{c}\text { Area } \\
\left(\mathrm{km}^{2}\right)\end{array}$ & & & $\begin{array}{l}\text { Rainy } \\
\text { season }\end{array}$ & $\begin{array}{c}\text { Dry } \\
\text { season }\end{array}$ & $\begin{array}{l}\text { Rainy } \\
\text { season }\end{array}$ & $\begin{array}{c}\text { Dry } \\
\text { season }\end{array}$ \\
\hline 1 & lake $^{2}$ & savannah & none & cloudy & underwater & 100 & 200 & 100 & 2 & 70 & 0.3 & 28 & 28.2 & 6.14 & 4.92 \\
\hline 2 & lake & savannah & none & clean & underwater & 100 & 80 & 80 & 6.4 & 60 & 0.15 & 29.2 & 32.3 & 5.80 & 6.33 \\
\hline 3 & lake & $\begin{array}{l}\text { savannah/ } \\
\text { forest }^{3}\end{array}$ & none & cloudy & floating & 150 & 100 & 100 & 10 & 100 & 0.15 & 31 & 31 & 5.90 & 5.38 \\
\hline 4 & lake $^{2}$ & savannah & none & cloudy & underwater & 200 & 30 & 35 & 1.05 & 50 & 0.20 & 26.5 & 28.5 & 5.70 & 5.23 \\
\hline 5 & lake $^{2}$ & savannah & weak & clean & underwater & 50 & 40 & 80 & 3.2 & 25 & 0.40 & 27.9 & 28 & 6.12 & 6.50 \\
\hline 6 & lake & savannah & none & cloudy & $\begin{array}{c}\text { floating/ } \\
\text { underwater }\end{array}$ & 50 & 250 & 500 & 125 & 90 & 0.30 & 28 & 29 & 5.60 & 5.20 \\
\hline 7 & cistern & $\begin{array}{l}\text { cultivated } \\
\text { savannah }\end{array}$ & none & cloudy & underwater & 100 & 18 & 35 & 0.063 & 15 & 0.20 & 29.2 & 29.3 & 5.80 & 6.46 \\
\hline 8 & lake $^{2}$ & $\begin{array}{l}\text { cultivated } \\
\text { savannah }^{4}\end{array}$ & none & cloudy & underwater & 150 & 10 & 60 & 0.6 & 25 & 0.40 & 31 & 30.4 & 6.30 & 6.24 \\
\hline 9 & lake & $\begin{array}{l}\text { savannah/ } \\
\text { forest }^{3}\end{array}$ & weak & clean & $\begin{array}{c}\text { floating/ } \\
\text { underwater }\end{array}$ & 400 & 1000 & 1000 & 1000 & 80 & 0.50 & 27.4 & 30.3 & 6.50 & 6.17 \\
\hline 10 & lake & $\begin{array}{l}\text { Seasonal forest/ } \\
\text { dense forest }{ }^{3}\end{array}$ & none & clean & underwater & 400 & 30 & 70 & 2.1 & 50 & 0.50 & 31 & 30.2 & 5.50 & 5.54 \\
\hline
\end{tabular}

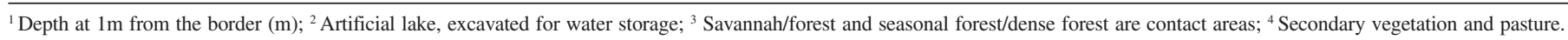

Affinity index between species: Affinity indices between pair of species had significant result $(\geq 0.544)$ for the pair An. triannulatus/ An. nuneztovari only (Tables 3 to 5). The pair An. triannulatus/An. nuneztovari and An. albitarsis/An. braziliensis were the pair of species that displayed the highest affinity indices (Table 5).

Diversity indices: Species diversity indices between breeding sites located in contact areas/cultivated savanna (sites 3, 7,8 and 10) and the savannah (sites 1, 2, 4, 5 and 6) were not statistically significant (Table 6). However, species diversity index was statistically significant higher for the dry season when compared to rainy season $\left(\mathrm{df}=7, \chi^{2}=\right.$ 60.32, $\mathrm{p} \geq 0.001$ ).

Meteorological data: During the rainy season maximum

Table 3

Anopheline species and number of specimens occurring in 10 breeding sites during the rainy and dry seasons, 2004-2005, Roraima, Brazil

\begin{tabular}{|c|c|c|c|c|c|c|c|c|c|c|}
\hline \multirow[t]{2}{*}{ Site Nb. } & \multirow[t]{2}{*}{ Season } & \multirow[b]{2}{*}{ albitarsis } & \multirow[b]{2}{*}{ triannulatus } & \multirow[b]{2}{*}{ braziliensis } & \multicolumn{2}{|c|}{ Species of Anopheles } & \multirow[b]{2}{*}{ darlingi } & \multirow[b]{2}{*}{ evansae } & \multirow[b]{2}{*}{ oswaldoi } & \multirow[b]{2}{*}{ Total } \\
\hline & & & & & nuneztovari & strodei & & & & \\
\hline 1 & Rainy & 18 & 0 & 1 & 0 & 0 & 0 & 0 & 0 & \\
\hline 2 & & 4 & 0 & 0 & 0 & 0 & 0 & 0 & 0 & \\
\hline 3 & & 1 & 0 & 0 & 0 & 0 & 0 & 0 & 0 & \\
\hline 4 & & 1 & 0 & 0 & 0 & 0 & 0 & 0 & 0 & \\
\hline 5 & & 24 & 0 & 32 & 0 & 0 & 0 & 0 & 0 & \\
\hline 6 & & 10 & 0 & 3 & 0 & 0 & 0 & 0 & 0 & \\
\hline 7 & & 0 & 46 & 1 & 52 & 0 & 0 & 1 & 2 & \\
\hline 8 & & 1 & 35 & 1 & 1 & 0 & 0 & 0 & 1 & \\
\hline 9 & & 4 & 0 & 1 & 0 & 0 & 0 & 0 & 0 & \\
\hline 10 & & 3 & 0 & 2 & 0 & 0 & 1 & 5 & 0 & \\
\hline 1 & Dry & 0 & 0 & 34 & 0 & 0 & 0 & 0 & 0 & \\
\hline 2 & & 0 & 0 & 16 & 0 & 0 & 0 & 0 & 0 & \\
\hline 3 & & 1 & 5 & 1 & 4 & 0 & 0 & 0 & 0 & \\
\hline 4 & & 1 & 0 & 34 & 4 & 0 & 0 & 0 & 0 & \\
\hline 5 & & 95 & 0 & 0 & 0 & 0 & 0 & 0 & 0 & \\
\hline 6 & & 13 & 0 & 0 & 0 & 0 & 0 & 0 & 0 & \\
\hline 7 & & 15 & 21 & 0 & 14 & 17 & 0 & 1 & 0 & \\
\hline 8 & & 0 & 37 & 0 & 14 & 10 & 0 & 0 & 0 & \\
\hline 9 & & 2 & 13 & 0 & 1 & 0 & 0 & 0 & 0 & \\
\hline 10 & & 0 & 0 & 0 & 1 & 9 & 7 & 0 & 2 & \\
\hline TOTAL & & 193 & 157 & 126 & 91 & 36 & 8 & 7 & 5 & 623 \\
\hline
\end{tabular}




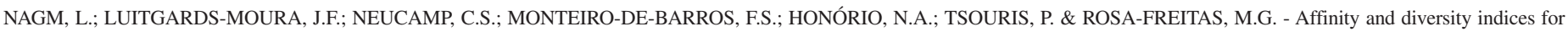
anopheline immature forms. Rev. Inst. Med. trop. S. Paulo, 49(5): 309-316, 2007.

Table 4

Single occurrence matrix for pairs of anophelines species in 10 breeding sites in both rainy and dry seasons (20 total possible occurrences)

\begin{tabular}{|c|c|c|c|c|c|c|c|c|}
\hline $\begin{array}{l}\text { Species of } \\
\text { Anopheles }\end{array}$ & albitarsis & triannulatus & braziliensis & Nuneztovari & strodei & Darlingi & evansae & oswaldoi \\
\hline albitarsis & & 2 & 3 & 3 & 2 & 1 & 1 & 2 \\
\hline triannulatus & 11 & & 8 & 2 & 1 & 2 & 1 & 1 \\
\hline braziliensis & 7 & 3 & & 4 & 3 & 1 & 1 & 1 \\
\hline nuneztovari & 10 & 0 & 7 & & 0 & 1 & 1 & 0 \\
\hline strodei & 14 & 4 & 11 & 5 & & 1 & 2 & 2 \\
\hline darlingi & 14 & 6 & 10 & 7 & 2 & & 2 & 2 \\
\hline evansae & 13 & 4 & 9 & 6 & 2 & 1 & & 2 \\
\hline oswaldoi & 14 & 4 & 9 & 5 & 2 & 1 & 2 & \\
\hline
\end{tabular}

Numbers showing above the diagonal bars are total number of occurrences for the species given in the first row alone, when paired to the species given in the first column. Numbers showing below the diagonal bars are total number of occurrences for the species given in the first column alone, when compared to the species given in the first row.

Table 5

Matrix of co-occurrence (above diagonal) and affinity index (below diagonal) between pair of anophelines species in 10 breeding sites pair of anopheline species

\begin{tabular}{|c|c|c|c|c|c|c|c|c|}
\hline $\begin{array}{l}\text { Species of } \\
\text { Anopheles }\end{array}$ & albitarsis & triannulatus & braziliensis & nuneztovari & strodei & darlingi & evansae & oswaldoi \\
\hline albitarsis & & 4 & 8 & 5 & 1 & 1 & 2 & 1 \\
\hline triannulatus & 0.218 & & 3 & 6 & 2 & - & 2 & 2 \\
\hline braziliensis & 0.494 & 0.165 & & 4 & - & 1 & 2 & 2 \\
\hline nuneztovari & 0.327 & 0.662 & 0.276 & & 3 & 1 & 2 & 3 \\
\hline strodei & 0.020 & 0.267 & - & 0.436 & & 1 & 1 & 1 \\
\hline darlingi & 0.053 & - & 0.062 & 0.073 & 0.120 & & 1 & 1 \\
\hline evansae & 0.169 & 0.183 & 0.197 & 0.231 & 0.045 & 0.055 & & 1 \\
\hline oswaldoi & 0.020 & 0.267 & 0.062 & 0.324 & 0.045 & 0.055 & 0.045 & \\
\hline
\end{tabular}

${ }^{1}$ Significant value in bold, i.e. $\geq 0.544$ corresponding to average +1 standard deviation $(0.350+0.194)$; - pair of species did not occur together.

temperature mean was $35.3{ }^{\circ} \mathrm{C}$ (varied from 34.6 to $36.5 \pm 0.6$ ), minimum temperature mean was $17.6{ }^{\circ} \mathrm{C}(13.2-22.3 \pm 3.5)$, rainfall mean $45.7 \mathrm{~mm}(17-83 \pm 23.9)$, available soil water mean $67 \%(43.4-$ $81.3 \pm 16.5)$. For the dry season values for maximum temperature mean was $35.7{ }^{\circ} \mathrm{C}(35.1-36.2 \pm 0.5)$, minimum temperature mean was 21.1 ${ }^{\circ} \mathrm{C}(18.9-24.1 \pm 1.9)$, rainfall mean $13.5 \mathrm{~mm}(0-31.7 \pm 15.4)$, available

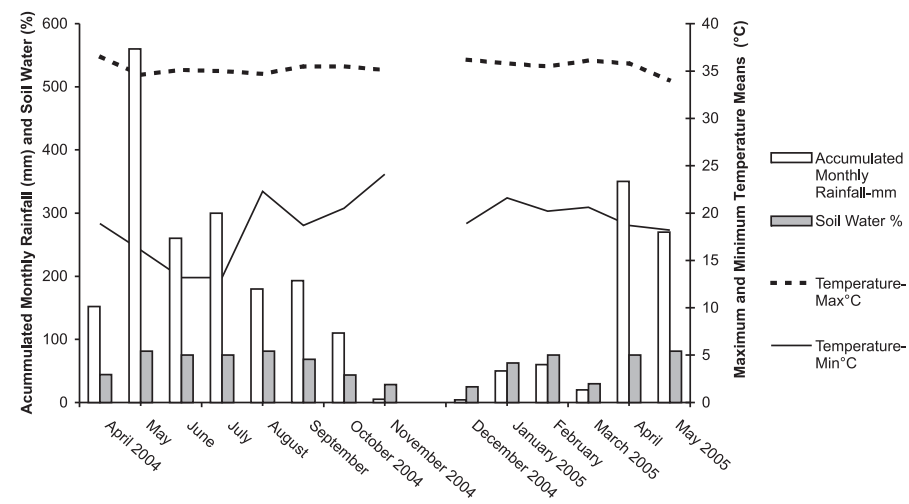

Fig. 3 - Meteorological variables prevailing in the study area depicted in Fig. 1. Rainy season is between April and November and dry season is from December to March. soil water mean $44.2 \%(24.9-75 \pm 23)$ (Fig. 3). Date of the last rain varied from zero to three days during the rainy season and from three to 24 days in the dry season.

\section{DISCUSSION}

Anopheline immature form collections were performed in permanent water reservoirs in the Boa Vista surroundings for its importance as most populated city and administrative center and, for logistic reasons. Cantá, in the Southeast border of Boa Vista, was the most malarigeneous municipality out of the 15 Roraima municipalities with API of 398.1 (2005) and 317.8 (2004), followed by Bonfim to the East ( $2^{\text {nd }}$ API in 2005 with 189.8 while held the $10^{\text {th }}$ API position in 2004 of 81.2). Alto Alegre, in the West border, presented an API of 67.7 in 2005 (65.5 in 2004). Boa Vista had an API of 32.6 in 2005 (25.1 in 2004). High malaria risk areas are those which API is $\geq 50$ according to Ministry of Health of Brazil. All localities where breeding sites were located reported malaria cases (Table 4).

Anophelines can exploit a broad range of water bodies as breeding places. Some species present a high ecological valence and not show preferences for a certain kind of breeding place while others are stricter on their choices. While An. albitarsis and An. braziliensis where 


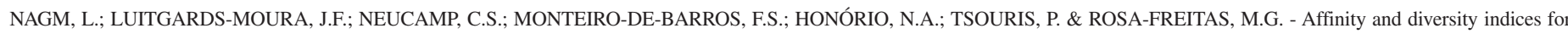
anopheline immature forms. Rev. Inst. Med. trop. S. Paulo, 49(5): 309-316, 2007.

Table 6

Shannon diversity index for 10 breeding sites for the rainy and dry seasons, 2004-2005, in Roraima, Brazil

\begin{tabular}{|c|c|c|c|c|c|c|c|c|c|c|}
\hline \multirow[b]{2}{*}{$\begin{array}{l}\text { Species of } \\
\text { Anopheles }\end{array}$} & \multicolumn{9}{|c|}{ Breeding site } & \multirow[b]{2}{*}{$\begin{array}{c}10 \\
\text { Seasonal forest/ } \\
\text { dense forest }\end{array}$} \\
\hline & $\begin{array}{c}1 \\
\text { savannah }\end{array}$ & $\begin{array}{c}2 \\
\text { savannah }\end{array}$ & $\begin{array}{c}3 \\
\text { savannah/ } \\
\text { forest }\end{array}$ & $\begin{array}{c}4 \\
\text { savannah }\end{array}$ & $\begin{array}{c}5 \\
\text { savannah }\end{array}$ & $\begin{array}{c}6 \\
\text { savannah }\end{array}$ & $\begin{array}{c}7 \\
\text { cultivated } \\
\text { savannah }\end{array}$ & $\begin{array}{c}8 \\
\text { cultivated } \\
\text { savannah }\end{array}$ & $\begin{array}{c}9 \\
\text { savannah/ } \\
\text { forest }\end{array}$ & \\
\hline \multicolumn{11}{|l|}{ Rainy season } \\
\hline albitarsis & -0.367 & -0.322 & -0.207 & -0.092 & -0.292 & -0.368 & 0 & -0.046 & -0.316 & -0.230 \\
\hline triannulatus & 0 & 0 & 0 & 0 & 0 & 0 & -0.354 & -0.367 & 0 & 0 \\
\hline braziliensis & -0.075 & 0 & 0 & 0 & -0.329 & -0.249 & -0.030 & -0.046 & -0.145 & -0.181 \\
\hline nuneztovari & 0 & 0 & 0 & 0 & 0 & 0 & -0.362 & -0.046 & 0 & 0 \\
\hline strodei & 0 & 0 & 0 & 0 & 0 & 0 & 0 & 0 & 0 & 0 \\
\hline darlingi & 0 & 0 & 0 & 0 & 0 & 0 & 0 & 0 & 0 & -0.113 \\
\hline evansae & 0 & 0 & 0 & 0 & 0 & 0 & -0.030 & 0 & 0 & -0.299 \\
\hline oswaldoi & 0 & 0 & 0 & 0 & 0 & 0 & -0.052 & -0.046 & 0 & 0 \\
\hline \multicolumn{11}{|l|}{ Dry season } \\
\hline albitarsis & 0 & 0 & -0.207 & -0.092 & -0.292 & -0.347 & -0.214 & 0 & -0.224 & 0 \\
\hline triannulatus & 0 & 0 & -0.365 & 0 & 0 & 0 & -0.258 & -0.368 & -0.297 & 0 \\
\hline braziliensis & -0.285 & -0.179 & -0.207 & -0.138 & 0 & 0 & 0 & 0 & 0 & 0 \\
\hline nuneztovari & 0 & 0 & -0.366 & -0.230 & 0 & 0 & -0.206 & -0.275 & -0.145 & -0.113 \\
\hline strodei & 0 & 0 & 0 & 0 & 0 & 0 & -0.230 & -0.230 & 0 & -0.361 \\
\hline darlingi & 0 & 0 & 0 & 0 & 0 & 0 & 0 & 0 & 0 & -0.340 \\
\hline evansae & 0 & 0 & 0 & 0 & 0 & 0 & -0.030 & 0 & 0 & 0 \\
\hline oswaldoi & 0 & 0 & 0 & 0 & 0 & 0 & 0 & 0 & 0 & -0.181 \\
\hline Shannon Rainy & 0.442 & 0.322 & 0.207 & 0.092 & 0.621 & 0.617 & 0.829 & 0.552 & 0.461 & 0.823 \\
\hline Shannon Dry & 0.285 & 0.179 & 1.145 & 0.461 & 0.292 & 0.347 & 0.939 & 0.873 & 0.666 & 0.995 \\
\hline
\end{tabular}

${ }^{1}$ Highest diversity indices in bold

collected in all 10 breeding sites with high specimens numbers in breeding sites located in the savannah, An. darlingi was found in breeding site 10 only, a shaded permanent water body in a savannah/ forest contact area, conditions known as ideal for its breeding ${ }^{4,6}$. FUNASA reports An. darlingi to be widely distributed all over the state. Nonetheless, An. darlingi was found in breeding site 10 only. This fact suggests that An. darlingi does not occur uniformly all over the region but presents limitation to specific landscapes.

The presence of some anopheline species could synergistically help other species. An. triannulatus/An. nuneztovari and An. albitarsis/An. braziliensis had the highest affinity indices (0.662 and 0.494, respectively) (Table 5). Even though the pair An. albitarsis/An. braziliensis was observed together in eight occasions rather than six occasions seen for the pair An. triannulatus/An. nuneztovari, the number of occasions each one of the species in both pairs were observed alone (Table 4) was decisive in the index outcome. High affinity index might indicate that species share preferences for the same breeding site conditions and/or that the presence of one species somehow facilitates the other. The same might happen at lower intensity for the pair of species An. nuneztovari/An. strodei and An. nuneztovari/An. oswaldoi.

Usually, contact areas are zones of ecological tension that tend to display higher plant diversity (CURTIS 1959 apud VAN DER MAAREL ${ }^{18}$ ). Whether plant diversity is related to anopheline diversity seen in those breeding sites is a subject that deserves further investigation. Although not statistically significant, disturbed areas represented by the cultivated savannah (breeding site 7 and 8) presented higher species diversity both during rainy and dry seasons (Table 6). Breeding site 7 in the cultivated savannah is a man-made excavation for rain water storage.

Higher species diversity was found for the dry season (Table 3). Dry season has been assumed to offer most stable environmental conditions. During rainy season rainfall values can reach $83 \mathrm{~mm}$ per day and it rains almost every day ${ }^{12}$. Accumulated rainfall of $560 \mathrm{~mm}$ per month was registered in May 2004 for Boa Vista ${ }^{12}$. In fact, during the time frame of our study, rainfall and soil water had the highest variance between the rainy and dry seasons. During the rainy season rainfall mean was $571.2 \mathrm{~mm}$ while this value decreased to $238.6 \mathrm{~mm}$ mean in the dry season. Interestingly, recent data shows that, contrary to the most of the vegetation around the world that follows a general pattern of growth and lush during the rainy season, the Amazon rainforest productivity is enhanced during the dry season ${ }^{11}$. In our view this indicates that environmental conditions are more stable during the dry season suitable for species to increase activity and population sizes.

Further taxonomical studies will help to understand species distributions for the three species complexes found (Albitarsis, Triannulatus and Oswaldoi). Recent studies show that An. albitarsis species $\mathrm{E}$ is the prevailing species in Boa Vista ${ }^{13}$. 


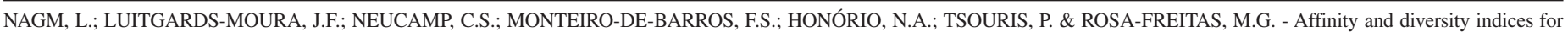
anopheline immature forms. Rev. Inst. Med. trop. S. Paulo, 49(5): 309-316, 2007.

An. darlingi breeding sites exist all over the Roraima territory most likely in patches of suitable ecological conditions for the species. Even though darlingi was found only in a forest breeding site (number 10) and in low numbers (one larva in the rainy season and seven larvae in the dry season) malaria case numbers remained the same throughout the period (Fig. 2). This finding is in accordance with low density numbers seen for $A n$. darlingi $i^{15}$. Natural infection investigations on adult anophelines in Boa Vista showed that An. darlingi even in low density is able to maintain malaria endemicity ${ }^{15}$.

Higher species diversity of anopheline species in contact areas of ecological tension, and anopheline species diversity boost in the dry season are fascinating issues that deserve attention as they are linked to malaria epidemiology. Further work in these areas might substantiate indications seen in this study and are under way.

\section{CONCLUSIONS}

An. braziliensis and An. albitarsis were the most frequently found species during the rain and dry seasons in savannah, savannah-forest and forest areas. Breeding sites in savannah-forest contact areas presented higher species diversity. An. darlingi was found in only one breeding site located in one of those savanna-forest contact areas. Even though An. darlingi is regarded as a mosquito of broad distribution in Roraima, it is most likely that the species is restricted to patches of suitable ecological conditions. An. triannulatus/An. nuneztovari and An. albitarsis/An. braziliensis were the pairs of species that mostly occurred together what might indicate a high compatibility for the same breeding conditions and/or a synergistic co-occurrence. Dry season displayed a higher number of specimens collected. This is in accordance with recent plant ecology studies ${ }^{11}$ that show an overall vegetation spur in the Amazon Forest during the dry season. An. darlingi larvae were found rarely. Nonetheless, malaria cases were reported throughout the study period. These larval observations corroborate previous adult data on anopheline Plasmodium infection ${ }^{15}$ that concluded that $A n$. darlingi specimens even in low numbers since accompanied by An. albitarsis are able to maintain malaria endemicity.

\section{RESUMO}

\section{Afinidade e diversidade de formas imaturas de anofelinos}

Como para toda a Amazônia, a malária representa um importante problema de saúde pública em Roraima. Roraima apresentou um índice parasitário anual de 85,4 em 2005, o maior do Brasil. O conhecimento dos criadouros de anofelinos constitui-se num componente primordial nas estratégias de controle da malária. No intuito de contribuir para o limitado conhecimento dos criadouros de anofelinos em Roraima, coleções de imaturos e observações dos criadouros foram realizadas no entorno da capital Boa Vista. As coletas foram feitas nas estações seca e chuvosa entre abril de 2004 e janeiro de 2005. Os criadouros observados foram reservatórios de água naturais e artificiais. Um total de 623 formas imaturas foram coletadas pertencentes às espécies $A n$. albitarsis s.l., An.triannulatus s.l., An. nuneztovari/dunhami, An. braziliensis, An. evansae, An. oswaldoi s.l., An. strodei e An. darlingi. An. braziliensis e An. albitarsis foram as espécies mais freqüentemente encontradas. Oito larvas de An. darlingi foram encontradas em apenas um criadouro na floresta. An. triannulatus/An. nuneztovari e An.
albitarsis/An. braziliensis foram os pares de espécies de maior coocorrência. Ambos pares de espécies apresentaram o maior índice de afinidade o que pode indicar compatibilidade para as mesmas condições prevalentes nos criadouros e/ou sinergismo. O índice de diversidade de espécies foi maior para a estação seca.

\section{REFERENCES}

1. AGRITEMPO - Sistema de monitoramento agrometeorológico. Available at http:// www.agritempo.gov.br. Accessed 23 October 2006.

2. ALLEN, M.J. \& MOORE, S.L. - 1996 Annual Report. Southern California Coastal Water Research Project. Recurrent Groups of Megabenthic Invertebrates on the Mainland Shelf of Southern California in 1994. Available at http:// www.sccwrp.org/pubs/annrpt/96/ar-14.htm

3. BARROS, F.S.M. - Diversidade e sazonalidade de Anopheles sp. (Diptera: Culicidae) com ênfase na prevalência de malária em Roraima. Recife, 2005. (Dissertação de Mestrado em Biologia Animal - Departamento de Zoologia, Universidade Federal de Pernambuco).

4. CONSOLI, R.A.G.B. \& OLIVEIRA, R.L. - Principais mosquitos de importância sanitária no Brasil. Rio de Janeiro, FIOCRUZ, 1994.

5. DE SOUZA, C.C. - Uso do hábitat por larvas de mosquitos anofelinos (Diptera; Culicidae) em áreas alteradas da região do Apiaú, município de Mucajaí, Roraima, Brasil. Manaus, 2003. (Dissertação de Mestrado em Ciências Biológicas/ Ecologia - Programa de Pós-graduação em Biologia Tropical e Recursos Naturais, Convênio Instituto Nacional de Pesquisas da Amazônia/Universidade Federal do Amazonas)

6. DEANE, L.M. - Malaria vectors in Brazil. Mem. Inst. Oswaldo Cruz, 81(supl. 2): 5-14, 1986.

7. FAGER, E. - Determination and analysis of recurrent groups. Ecology, 38: 586-595, 1957.

8. FAGER, E.W. \& McGOWAN, J.A. - Zooplankton species groups in the North Pacific. Science, 140: 453-461, 1963

9. FUNASA. FUNDAÇÃO NACIONAL DE SAÚDE - Vigilância Epidemiológica 2002. Programa Nacional de Controle da Malária. Available at http:// dtr2001.saude.gov.br/svs/epi/malaria/ pncm.htm. Accessed 21 April 2006.

10. GORHAM, J.R.; STOJANOVIC, C.J. \& SCOTT, H.G. - Illustrated key to the anopheline mosquitoes of Western South America. Mosquito Systematics, 5(2): 97-156, 1973 Available at http://wrbu.si.edu/www/MS/05/ MS05N02P097.PDF. Accessed 05 July 2006.

11. HUETE, A.R.; DIDAN, K.; SHIMABUKURO, Y.E. et al. - Amazon rainforests green-up with sunlight in dry season. Geophys. Res. Lett., 33: L06405, 2006.

12. INSTITUTO NACIONAL DE METEOROLOGIA - Gráficos das condições registradas. Available at http://www.inmet.gov.br/html/observacoes.php. Accessed 12 March 2006.

13. PÓVOA, M.M.; SOUZA, R.T.L.; LACERDA, R.N.L. et al. -The importance of Anopheles albitarsis $\mathrm{E}$ and An. darlingi in human malaria transmission in Boa Vista, State of Roraima, Brazil. Mem. Inst. Oswaldo Cruz, 101: 163-168, 2006.

14. SHANNON, C.E. - A mathematical theory of communications. Bell Syst. techn. J., 27: 379-423, 623-656, 1948

15. SILVA-VASCONCELOS, A.; KATÓ, M.Y.N.; MOURÃO, E.N. et al. - Biting indices, host-seeking activity and natural infection rates of Anopheline species in Boa Vista, Roraima, Brazil from 1996 to 1998. Mem. Inst. Oswaldo Cruz, 97: 151-161, 2002. 
NAGM, L.; LUITGARDS-MOURA, J.F.; NEUCAMP, C.S.; MONTEIRO-DE-BARROS, F.S.; HONÓRIO, N.A.; TSOURIS, P. \& ROSA-FREITAS, M.G. - Affinity and diversity indices for anopheline immature forms. Rev. Inst. Med. trop. S. Paulo, 49(5): 309-316, 2007.

16. SMITH, J.L. \& K. HYRENBACH, D. - Galápagos islands to British Columbia: seabird communities along a $9000 \mathrm{~km}$ transect from the tropical to the subarctic eastern Pacific ocean. Marine Ornithology, 31: 155-166, 2003.

17. TADEI, W.P.; THATCHER, B.D.; SANTOS, J.M. et al. - Ecologic observations on anopheline vectors of malaria in the Brazilian Amazon. Amer. J. trop. Med. Hyg., 59: $325-335,1998$.
18. VAN DER MAAREL, E. - Ecotones and ecoclines are different. J. Vegetation Sci., 1: 135-138, 1990.

19. VENRICK, E.L. - Phytoplankton in an oligotrophic ocean: observations and questions. Ecological Monographs, 52: 129-154, 1982.

Received: 26 April 2006

Accepted: 9 April 2007 